\title{
BRAGANTIA
}

Revista Científica do Instituto Agronômico, Campinas

Vol. 38

Campinas, dezembro de 1979

N.० 23

\section{"DELINEAMENTO DUPLO CENTRAL COMPOSTO COM 29 PONTOS"}

Armando Conagin e Joassy de Paul a Neves Jorge $\left({ }^{2}\right)$, Divisão de Plantas Alimenticias Básicas, Instituto Agronômico

\section{SINOPSE}

O delineamento duplo central composto com 29 pontos representa uma extensão do delineamento central composto e foi desenvolvido para o estudo de três fatores em mais de três níveis,

Essencialmente, consta de dois fatoriais $2^{3}$ (niveis 1 e $\mathrm{B}$ ), de duas estrelas (níveis $\alpha$ e $2 \alpha$ ) e de um ponto no centro do delineamento.

E mostrada a origem do delineamento, o tipo não ortogonal, completamente casualizado, o tipo ortogonal completamente casualizado e o ortogonal divisivel em dois blocos.

No primeiro deles, seriam estudados três fatores em cinco niveis $(-2,-1,0$. $+1 \mathrm{e}+2)$; no segundo, três fatores em nove niveis $(-3,02 ;-2,00 ;-1,51 ;-1,00 ; 0,00$; $+1,00 ;+1,51 ;+2,00 \mathrm{e}+3.02)$ e, no terceiro, três fatores nos nove niveis seguintes $(-7,262 ;-4,391 ;-3,631 ;-1,000 ; 0,000 ;+1,000 ;+3,631 ;+4,391$ e $+7,262)$.

E a rresentado também um exemplo do último delineamento, com sua análise respectiva.

De asordo com o critério de Box \& Wilson (2), esse último delineamento è mais eficiente que o fatorial $3 \times 3 \times 3$ divisivel em blocos de nove.

Os três delineamentos podem ser empregados em programas de adubaçāo visando ao estudo de macronutrientes com vistas à recomendação das doses ótimas de fertilizantes que possibilitem a maximização do lucro obtido e, ainda, em outras áreas da pesquisa científica em que se deseja a avaliação da superfície de resposta, o estudo dos seus pontos extremos, etc.

\section{INTRODUÇÃO}

Em programas de pesquisa com fertilizantes, utilizaram-se no passado experimentos simples, variando algumas fórmulas; mais tarde, usaram-se os ensaios fatoriais $2 \times 2 \times 2$; para o estudo de três fatores, em mais de

(1) Trabalho apresentado na Reunião da Região Brasileira da Sociedade Internacional de Bio. metria, realizada em 30 de março de 1978 , em comemorição ao $900^{\circ}$ aniversário do I.A. Recebido para publicação em 10 de setembro de 1979.

(*) Com bolsa de suplementação do $\mathrm{CNPa}$ 
dois níveis, o delineamento mais empregado foi, seguramente, o $3 \times 3 \times 3$, em blocos de nove. Programas experimentais de adubação com esse delineamento foram feitos na Inglaterra com bons resultados, principalmente com a beterraba açucareira; os $3 \times 3 \times 3$ foram intensivamente utilizados na India, além de experimentos simples, em arroz, trigo e milho etc.; na China (12), para programas com algodão, milho, trigo, arroz e colza; em Quênia, para pesquisa de cereais; no Brasil, nas pesquisas de adubação de cana-de-açúcar $(\mathbf{1}, \mathbf{7}, \mathbf{1 3})$, algodão (5), milho $(9,11,15)$, feijão (6) e amendoim (14).

O aparecimento de "pontos de sela", quando da análise dos experimentos individuais e mesmo de grupos de experimentos, levou pesquisadores em busca de outros delineamentos, principalmente os do tipo Box $(4,16) \mathrm{e}$, em alguns casos, os fatoriais fracionados (3).

Um delineamento do tipo central composto, muito utilizado na experimsntação industrial, é o $2^{3}+2(3)+\mathrm{n}_{1}$. Além do cubo central nos níveis -1 e +1 para três fatores, dispõe de dois pontos axiais nas distâncias $-\alpha c-\alpha$ para cada fator (ná dose média dos outros dois fatores) e, ainda, de um ponto central 000 a ser repetido $n_{1}$ vezes para possibilitar a avaliação do erro experimental e a adequação do modelo (2).

Na experimentação agronômica foi utilizado, entre nós, esse delineamento com o valor $\alpha=-2$ e $\alpha=+2$. tendo-se usado duas repetições do mesmo (8). Com $\alpha$ convenientemente escolhido, pode-se obter o delineamento central composto ortogonal, que facilita a obtenção das estimativas dos coeficientes lineares, quadráticos e interações $(\mathbf{1 0})$.

Os autores, visando obter melhor estimativa da curvatura da função de resposta, propuseram, em 1968, o uso do delincamento duplo central composto com 29 pontos (4).

\section{MATERIAL E METODO}

Esse delneamento em sua forma original era composto de dois cubos situados nas distâncias $\pm 1 \mathrm{e}^{ \pm 2}$, duas estrelas nas distáncias $\alpha= \pm 1$ e $2 \alpha=$ 2 e um ponto central.

O delineamento pode ser representado por $2^{*}+2^{*}+2(3)+2(3)+1$ $\therefore$ geometricamente, tem a forma ilustrada na figura 1 .

Mais ou monos na mesma época, e, independentemente, havia sido proposto por Voss \& Pesek (16) um delineamento da mesma natureza, só diferindo por apresentar seis pontos em vez de doze, na estrela, num total de 23 pontos. 


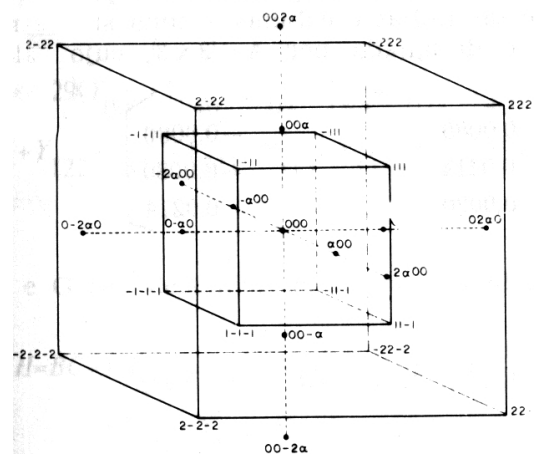

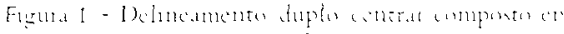

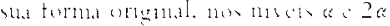

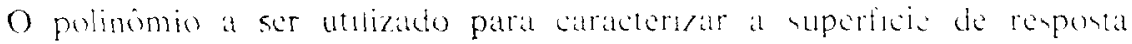
é de naturezar quadrática:

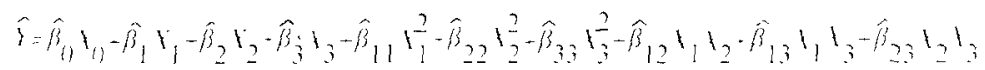

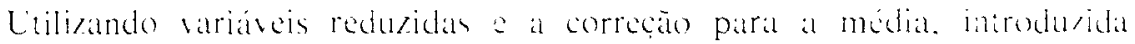
nos termos quadritioss. chega-se an polinomio:

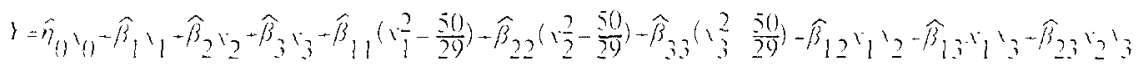

$O$ vetor $\mathbf{Y}$ das produções ${ }_{i j k}$, correspondentes aos tratamentos numerados de 1 a 29 , e a matriz $X$ das variáveis independentes, dadas em unidades reduzidas, são mostrados na página 220.

A matriz $\mathrm{A}=\mathrm{X} X$, apresentada abaixo, é idêntica à do referido trabalho, tendo, porém, sido reordenada no sentido de colocar os termos correspondentes aos coeficientes lineares em primeiro lugar, seguidos dos coeficientes quadráticos $\mathfrak{e}$ dos correspondentes às interações.

$\mathbf{A}=\mathrm{X} \cdot \mathrm{X}=\left[\begin{array}{rrrrrccccc}29 & 0 & 0 & 0 & 0 & 0 & 0 & 0 & 0 & 0 \\ 0 & 50 & 0 & 0 & 0 & 0 & 0 & 0 & 0 & 0 \\ 0 & 0 & 50 & 0 & 0 & 0 & 0 & 0 & 0 & 0 \\ 0 & 0 & 0 & 50 & 0 & 0 & 0 & 0 & 0 & 0 \\ 0 & 0 & 0 & 0 & 2430.29 & 144429 & 1444 \cdot 29 & 0 & 0 & 0 \\ 0 & 0 & 0 & 0 & 144429 & 2430.29 & 144429 & 0 & 0 & 0 \\ 0 & 0 & 0 & 0 & 1444.29 & 144429 & 2430 \cdot 29 & 0 & 0 & 0 \\ 0 & 0 & 0 & 0 & 0 & 0 & 0 & 136 & 0 & 0 \\ 0 & 0 & 0 & 0 & 0 & 0 & 0 & 0 & 136 & 0 \\ 0 & 0 & 0 & 0 & 0 & 0 & 0 & 0 & 0 & 136\end{array}\right]$


A matriz $\bar{A}^{-1}(10 \times 10)$ contém os recíprocos dos valores correspondentes à parte que é diagonal, com zeros para os demais elementos dessas linhas e colunas, e uma submatriz $3 \times 3$, que corresponde aos termos quadráticos e é designada por $\mathrm{A}^{-1}(3 \times 3)$, cujo valor numérico é transcrito a seguir:

$\mathrm{A}^{-1}(3 \times 3)=\left[\begin{array}{rrr}0,0214 & -0,0080 & -0,0080 \\ -0,0080 & 0,0214 & -0,0080 \\ -0,0080 & -0,0080 & 0,0214\end{array}\right]$

Sabemos que:

$\underline{\hat{\beta}}=\mathrm{A}^{-1} \mathrm{X}^{\mathrm{Y}} \underline{\mathrm{Y}}$, onde:

$\overline{\mathrm{X}}^{\prime} \underline{\mathrm{Y}}=[\mathrm{A}, \mathrm{B}, \mathrm{C}, \ldots, \mathrm{I}, \mathrm{J}]$, com:

$A=\sum_{1}^{29} Y_{i j k}$

$$
\begin{aligned}
B= & -2\left(Y_{000}+Y_{040}+Y_{004}+Y_{044}+Y_{022}\right)-1\left(Y_{111}+Y_{131}+Y_{113}+Y_{133}+Y_{122}\right)+1\left(Y_{311}+Y_{331}+Y_{313}+Y_{333}+\right. \\
& \left.+Y_{322}\right)+2\left(Y_{400}+Y_{440}+Y_{404}+Y_{444}+Y_{422}\right) \\
& x_{0} x_{1} x_{2} x_{3}\left(x_{1}^{2}-50 / 29\right)\left(x_{2}^{2}-50 / 29\right)
\end{aligned}
$$

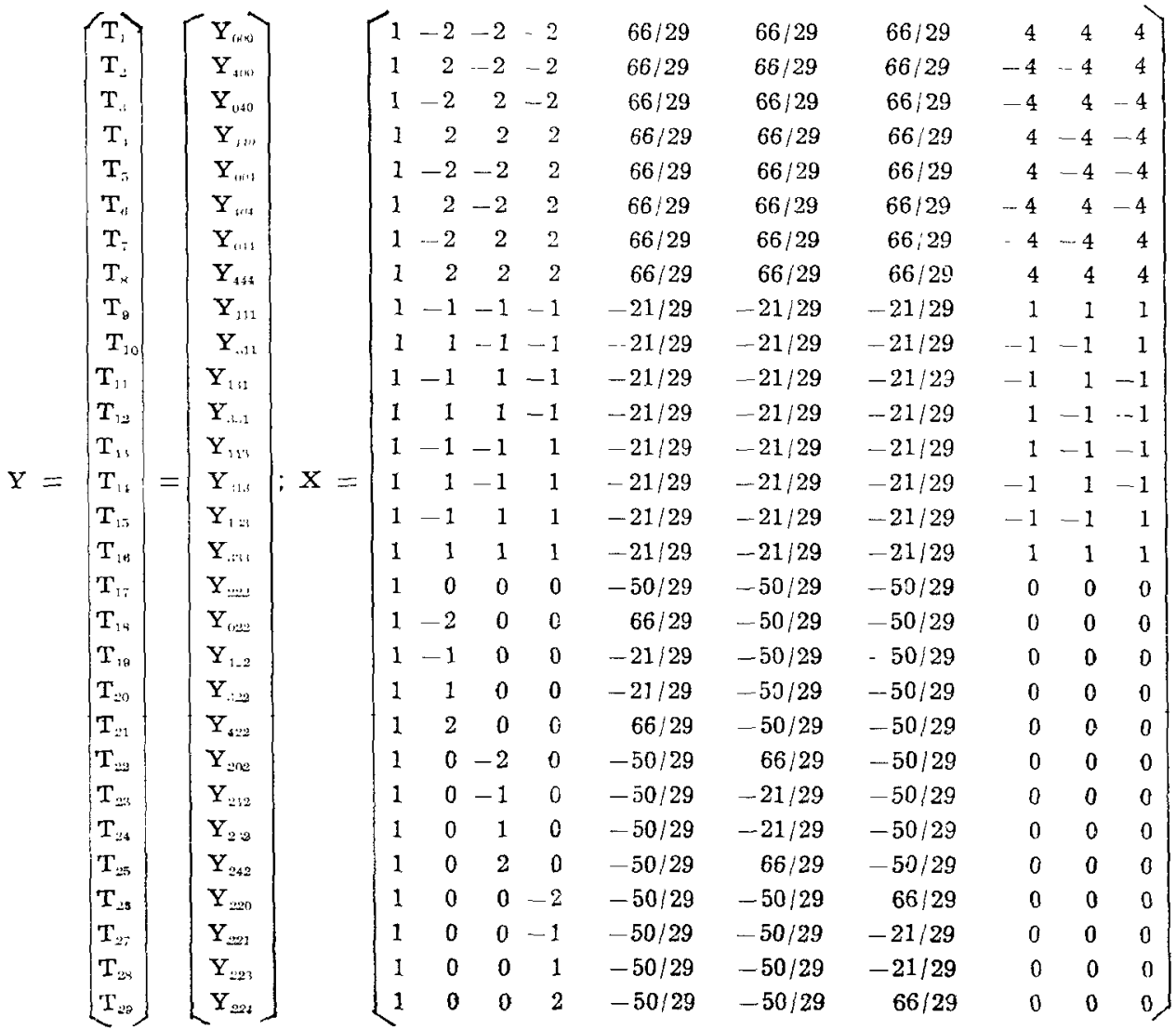




\section{De 2,1979 DELINEAMENTO DUPLO CENTRAL COMPOSTO \\ C e D são definidos de forma análoga. conforme explicito na matriz $\mathrm{X}$.}

$$
\begin{aligned}
E= & =(66 / 29)\left(Y_{000}+Y_{040}+Y_{004}+Y_{044}+Y_{022}+Y_{400}+Y_{422}+Y_{440}+Y_{404}+Y_{444}\right)-(21 / 29)\left(Y_{111}+Y_{131}+Y_{113}+Y_{133}+\right. \\
& \left.+Y_{122}+Y_{311}+Y_{331}+Y_{313}+Y_{333}+Y_{322}\right)-(50 / 29)\left(Y_{222}+Y_{202}+Y_{212}+Y_{232}+Y_{242}+Y_{220}+Y_{221}+Y_{223}+Y_{224}\right)
\end{aligned}
$$

$F$ e $G$ săo definidos de forma semelhante

$$
\begin{aligned}
& H=B C=4\left(Y_{000}+Y_{004}+Y_{440}+Y_{444}\right)+1\left(Y_{111}+Y_{113}+Y_{331}+Y_{333}\right)-4\left(Y_{400}+Y_{404}+Y_{040}+Y_{044}\right)-1\left(Y_{311^{+}}\right. \\
& \left.+Y_{313}+Y_{131}+Y_{133}\right) \\
& I=B D=4\left(Y_{000}+Y_{040}+Y_{404}+Y_{444}\right)+1\left(Y_{111}+Y_{131}+Y_{313}+Y_{333}\right)-4\left(Y_{440}+Y_{400}+Y_{004}+Y_{044}\right)-1\left(Y_{311^{+}}\right. \\
& \left.+Y_{331}+Y_{113}+Y_{133}\right) \\
& J=C D=4\left(Y_{000}+Y_{400}+Y_{044}+Y_{444}\right)+1\left(Y_{111}+Y_{311}+Y_{133}+Y_{333}\right)-4\left(Y_{040}+Y_{440}+Y_{004}+Y_{404}\right)-1\left(Y_{131}+\right. \\
& \left.+Y_{331}+Y_{113}+Y_{313}\right) \\
& \underline{\hat{\beta}}=A^{-1} \mathbf{X} \underline{\mathbf{Y}}= \\
& \left(\begin{array}{c}
\hat{\eta}_{n} \\
\widehat{\beta}_{1} \\
\widehat{\beta}_{:} \\
\widehat{\beta}_{:} \\
\hat{\beta}_{1:} \\
\widehat{\beta}_{2:} \\
\widehat{\beta}_{1:} \\
\widehat{\beta}_{1:} \\
\widehat{\beta}_{13} \\
\hat{\beta}_{2:}
\end{array}\right)
\end{aligned}
$$

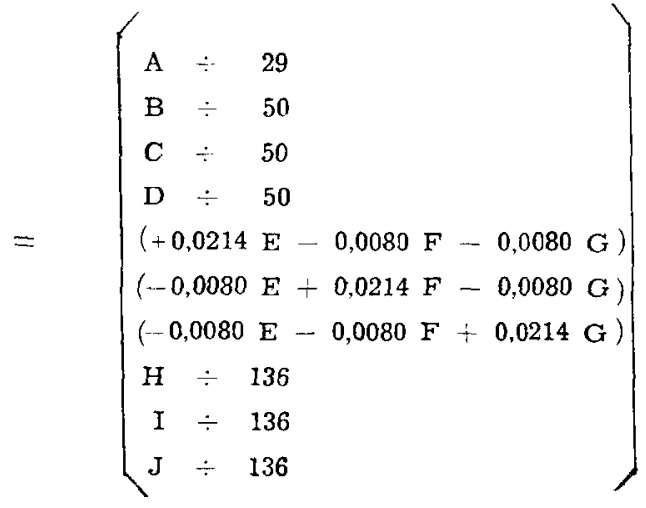

A soma de quadrados é dada por: $\underline{\hat{\beta}}{ }^{\prime} \mathbf{X}^{\prime} \mathbf{Y}$ $S Q \hat{\eta}_{0}=\frac{1}{29}(A)^{2} ; S Q \hat{\beta}_{1}=\frac{1}{50}(B)^{2} ; S Q \hat{\beta}_{12}=\frac{1}{136}(H)^{2}$ etc.

$S Q\left[\hat{\beta}_{11} \widehat{\beta}_{22}, \widehat{\beta}_{33}\right]=\{[0,0214 E-0,0080 F-0,0080 G] E+[-0,0080 E+0,0214 F-0,0080 G] F+[-0,0080 E-$ $-0,0080 F+0,0214 G] G$ 
Os componentes lineares e as interaçōes são postos em prova independentemente. A soma de quadrados correspondente aos coeficientes quadráticos é avaliada englobadamente, e esses coeficientes são testados em conjunto:

$$
S Q\left[\widehat{\beta}_{11}, \hat{\beta}_{22}, \hat{\beta}_{33}\right] \div 3
$$

O resíduo apresenta 19 graus de liberdade.

Vimos, no trabalho inicial (4), que:

$$
\hat{\eta}_{0}=\widehat{\beta}_{0}+\frac{50}{29}\left(\hat{\beta}_{11}+\hat{\beta}_{22}+\widehat{\beta}_{33}\right)
$$

Entāo:

$$
\hat{\beta}_{0}=\hat{\eta}_{0}-\frac{50}{29}\left(\hat{\beta}_{11}+\hat{\beta}_{22}+\hat{\beta}_{33}\right)
$$

As variâncias e covariâncias dos coeficientes de regressão são as seguintes:

$$
\begin{array}{rlrl}
v \hat{\beta}_{i}=1 / 50 \sigma^{2} & \widehat{V}_{i}=1 / 50 s^{2} & i=1,2.3 \\
v \widehat{\beta}_{i j}=1 / 136 \sigma^{2} & \widehat{V}_{i j}=1 / 136 s^{2} & i \neq j=1,2,3 \\
v \widehat{\beta}_{i i}=0,0214 \sigma^{2} & \widehat{V}_{i i}=0,0214 s^{2} & i \\
\operatorname{Cov} \widehat{\beta}_{11} \widehat{\beta}_{22}=-0,0080 \sigma^{2} & \operatorname{Cov} \widehat{\beta}_{11} \hat{\beta}_{22}=-0,0080 \mathrm{~s}^{2}, \text { etc. } &
\end{array}
$$

Um exemplo completo com a correspondente análise da variância encontra-se no trabalho citado (4).

\subsection{DUPLO CENTRAL COMPOSTO ORTOGONAL}

O uso de delineamentos ortogonais leva à obtenção de uma matriz A diagonal, o que facilita a estimação dos parâmetros e a análise da variancia correspondente.

$O$ vetor $\underline{Y}$ das observações contém 29 termos; a matriz $D$ do delineamento é semelhante à atrás considerada (constituída pela segunda, terceira e quarta colunas de $\mathrm{X}$ ), com a diferença de se ter um valor $\alpha$ genérico no lugar de $\alpha=1$ usado no delineamento anterior (tratamentos $Y_{18}, Y_{19}, \ldots, Y_{29}$ ).

A matriz $\mathrm{A}=\mathrm{X}^{\prime} \mathrm{X}$ para esse $\alpha$ genérico, com os termos quadráticos corrigidos para a média, onde a correção é $\mathrm{c}=10\left(4+\alpha^{2}\right) \div 29$, é dada por:

$\hat{\mathbf{A}}=\left[\begin{array}{cccccccccc}29 & 0 & 0 & 0 & 0 & 0 & 0 & 0 & 0 & 0 \\ 0 & 40+10 \alpha^{2} & 0 & 0 & 0 & 0 & 0 & 0 & 0 & 0 \\ 0 & 0 & 40+10 \alpha^{2} & 0 & 0 & 0 & 0 & 0 & 0 & 0 \\ 0 & 0 & 0 & 40+10 \alpha^{2} & 0 & 0 & 0 & 0 & 0 & 0 \\ 0 & 0 & 0 & 0 & \mathrm{p} & \mathrm{q} & \mathrm{q} & 0 & 0 & 0 \\ 0 & 0 & 0 & 0 & \mathrm{q} & \mathrm{p} & \mathrm{q} & 0 & 0 & 0 \\ 0 & 0 & 0 & 0 & \mathrm{q} & \mathrm{q} & \mathrm{p} & 0 & 0 & 0 \\ 0 & 0 & 0 & 0 & 0 & 0 & 0 & 136 & 0 & 0 \\ 0 & 0 & 0 & 0 & 0 & 0 & 0 & 0 & 136 & 0 \\ 0 & 0 & 0 & 0 & 0 & 0 & 0 & 0 & 0 & 136\end{array}\right]$


onde:

$$
\begin{aligned}
& p=F(4-c)^{2}+F(1-c)^{2}+2\left(\alpha^{2}-c\right)\left(\alpha^{2}-c\right)+2\left(4 \alpha^{2}-c\right)\left(4 \alpha^{2}-c\right)+(T-4) c^{2} \\
& q=F(4-c)^{2}+F(1-c)^{2}-4 c\left(4 \alpha^{2}-c\right)-4 c\left(\alpha^{2}-c\right)+(T-8) c^{2}
\end{aligned}
$$

onde $F=2^{3}=8$ e $T=2(3)+2(3)+1=13$

Efetuando as operaçōes e simplificando, chega-se a que $p=q+34 \alpha^{4}$ (10).

A partir da equação em q, obtém-se:

$q=136+29 c^{2}-80 c-20 c \alpha^{2}$

Substituindo c pelo seu valor, fazendo $\alpha^{2}=\mathrm{z}$ e lembrando que, para a matriz ficar ortogonal, deve-se ter $q=0$, chega-se à equação $z^{3}+8 z-23,44=0$, cuja raiz válida é a positiva.

$\mathrm{z}=\alpha^{2}=2,2801$, de onde $\alpha=1,5100$. Portanto, usando $\alpha=1,5100 \mathrm{em}$ lugar de $\alpha=1,00$, obter-se-á o delineamento duplo central composto ortogonal.

$O$ vetor $\underline{\mathbf{Y}}$ e a matriz $\mathrm{D}$ desse delineamento encontram-se na página 224 .

A matriz $\mathbf{A}=\mathrm{X}^{\prime} \mathrm{X}$ é diagonal $\mathrm{e}$ tem os seguintes valores:

$$
a_{11}=29 ; \quad a_{22}=a_{33}=a_{44}=62,8010 ; \quad a_{55}=a_{66}=a_{77}=176,7623 ; a_{88}=a_{99}=a_{10,10}=136 .
$$

A matriz $\mathrm{A}^{-1}$ é composta dos inversos, isto é:

$$
c_{11}=a_{11}^{-1}=1 / 29=0,034483 ; \quad c_{22}=a_{22}^{-1}=0,015923 ; \quad c_{55}=a_{55}^{-1}=0,005657 ; \quad c_{88}=a_{88}^{-1}=0,007353
$$

Com a ortogonalizaçăo obtida, a análise da variância fica grandemente facilitada, sendo os coeficientes $\hat{\eta}_{0}, \widehat{\beta}_{1}, \widehat{\beta}_{11}$ e $\hat{\beta}_{1}$, estimados diretamente, com as variâncias abaixo:

$$
\begin{array}{ll}
V \hat{\eta}_{0}=\sigma^{2} / 29 & \hat{V} \hat{\eta}_{0}=s^{2} / 29 \\
V \hat{\beta}_{i}=\sigma^{2} / 62,8010 & \hat{V} \hat{\beta}_{i}=s^{2} / 62,8010 \\
V \hat{\beta}_{i i}=\sigma^{2} / 176,7623 & \hat{V} \hat{\beta}_{i i}=s^{2} / 176,7623 \\
V \hat{\beta}_{i j}=\sigma^{2} / 136 & \hat{V}_{i j}=s^{2} / 136 \\
\operatorname{Cov} \hat{\beta}_{i} \hat{\beta}_{j}=\operatorname{Cov} \hat{\beta}_{i} \hat{\beta}_{i i}=\operatorname{Cov} \widehat{\beta}_{i} \hat{\beta}_{i j}=\operatorname{Cov} \hat{\beta}_{i i} \hat{\beta}_{i j}=\operatorname{Cov} \hat{\eta}_{0} \hat{\beta}_{i}=0 \text { etc. }
\end{array}
$$




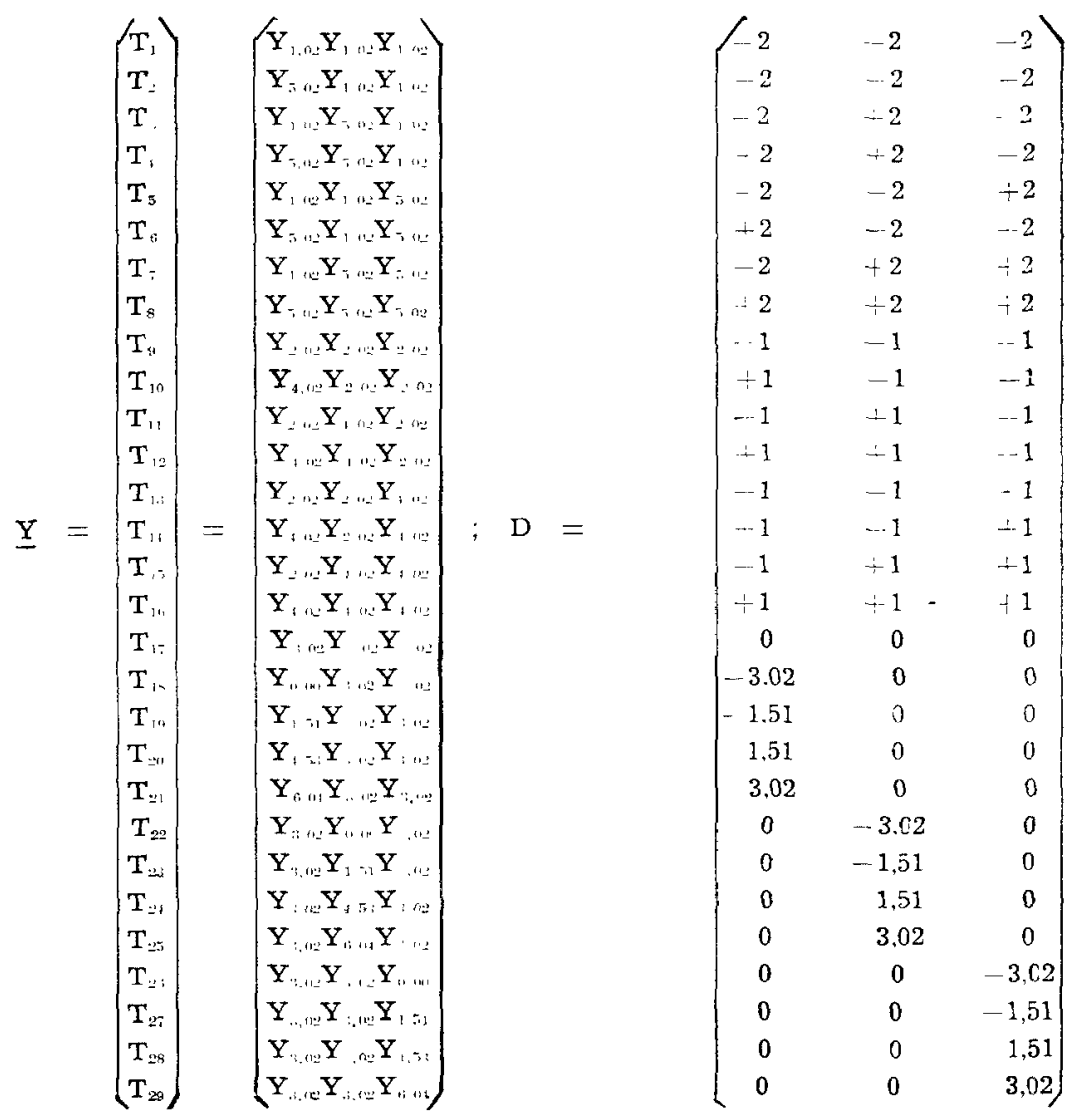

2.2 DELINEAMENTO DUPLO CENTRAL COMPOSTO COM 29 PONTOS, EM DOIS BLOCOS

O modelo completo, incluindo a partição em blocos, é o que se segue:

$$
\begin{gathered}
Y_{u}=\beta_{0}+\sum_{i=l}^{3} \beta_{i} x_{i u}+\sum_{i=l}^{3} \beta_{i i} x_{i u}^{2}+\sum_{i} \sum_{j} \beta_{i<j} \beta_{i j} x_{i u} x_{j u}+\sum_{m=l}^{b} \delta_{m}\left(z_{m u}-\tilde{z}_{m}\right)+\varepsilon u^{(u=1,2, \ldots N)} \\
\text { onde } x_{i u}^{2}=\left(x_{i u}^{2} \frac{\sum x_{i u}^{2}}{29}\right) .
\end{gathered}
$$

No caso, $z_{\text {tau }}$ será igual a 1 quando a observação u se encontrar no bloco considerado, e zero em caso contrário; por definiçāo, $\bar{z}_{m}$ é a fraçāo do total de observaçōes que estão no bloco $\mathrm{m}$. 
Para haver ortogonalitade entre os blocos e os outros componentes do modelo, deve-se ter:

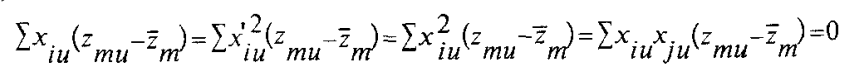

Para o delineamento proposto, são verificadas:

$$
\sum x_{i u}=\sum x_{i u} x_{j u}=0
$$

Se associarmos essas relaçōes, serāo duas as condiçōes que devem ser consideradas, dentro de cada bloco, para a formação de blocos ortogonais. A primeira delas é:

$$
\begin{aligned}
& b l \cdot m x_{i u}=0,(m=1,2 ; \quad i j=1,2,3 ; \quad i \neq j) \\
& \sum_{b l \cdot m} x_{i u} x_{j u}=0,(m=1,2)
\end{aligned}
$$

A segunda decorre de

$$
\sum x_{i u}^{2}\left(z_{m u}-\bar{z}_{m}\right)=0, o u: \quad \sum_{u=i}^{N} x_{i u m u}^{2}=\bar{z}_{m} \sum_{u=i}^{N} x_{i u}^{2}
$$

o que implica em que: "a contribuiçăo de determinado bloco para a soma total do quadrado da variável $x_{1}$ (a contribuição para $\sum x_{i u}^{2}$ ) é proporcional ao número də
observaçós existentes no bloco" (10).

Se se colocar a parte fatorial do delineamento em um dos blocos e a parte axial ccm o centro, no outro, a primeira condiçāo de ortogonalidade é verificada, sendo independente do valor de $\alpha$ considerado.

Para que a segunda condição se verifique, deve-se ter:

$$
\frac{\sum x_{i u}^{2}(\text { axial e centro })}{\sum x_{i u}^{2}(\text { fatorial })}=\frac{2 k+2 k+1}{2^{k}+2^{k}} ; \frac{10 \alpha^{2}}{8\left(1+\beta^{2}\right)}=\frac{12+1}{16}=0,8125 .
$$

Para isso, é preciso conciliar as condiçōes de ortogonalidade da matriz $(q=0)$ com as de ortogonalidade para blocos e passar a admitir os valores $\alpha, 2 \alpha, 1$ e $\beta$ respectivamente como niveis para as porções axiais e fatoriais do delineamento. Resolvendo, chega-se a $\alpha=3,6308$ e $\beta=4,3911$.

E fácil ver que: $\frac{10 \alpha^{2}}{8\left(1+\beta^{2}\right)}=\frac{12+1}{16}=0,8125$

O delineamento obtido possui os nove niveis seguintes: $-7,261 ;-4,391 ;-3,631$; $-1,000 ; 0 ;+1,000 ;+3,631 ;+4,391$ e $+7,261$.

Esses niveis, em termos de uma escala crescente a partir do valor zero, sāo os seguintes: $0 ; 2,871 ; 3,631 ; 5,262 ; 7,262 ; 8,262 ; 10,893 ; 11,653$ e 14,524 .

A matriz $D$ do delineamento é composta das colunas 2,3 e 4 da matriz $X$, transcrita na página 227. Devido à restriçāo de que a soma dos blocos deve ser nula, deve-se fazer a reparametrizaçāo $\delta,=-\delta z=\delta$ : a matrk $X$ passará a ter onze colunas em vez de doze. 
A matriz $\mathbf{A}=\mathbf{X}^{\prime} \mathbf{X}$ terá os seguintes componentes:

$a_{11}=29 ; \quad a_{22}=a \overline{\overline{33}} a_{44}=294,0886 ; \quad a_{55} a_{66}=a_{77}=5909,6165 ; \quad a_{88}=a_{99}=a_{10,10}=2982,0248 ;$

$a_{11,11}=7,1724$

Os termos $a_{11}$, para $i \neq j$, são iguais a zero.

A matriz inversa $\mathrm{A}^{-1}$ tem os seguintes valores:

$a_{11}^{-1}=c_{11}=0,034483 ; \quad c_{22}=c_{33}=c_{44}=0,003400 ; \quad c_{55}=c_{66}=c_{77}=0,000169 ; c_{88}=c_{99}=c_{10,10}=0,000335$; $c_{11,11}=0,139423$

A partir da matriz $A^{-1}$ e do vetor $X^{\prime} \underline{Y}$, calcula-se: $\underline{\hat{\beta}}=A^{-1} \mathbf{X}^{\prime} \underline{\underline{X}}$. Tem-se:

$\underline{X}^{\prime} \underline{Y}=\left[\begin{array}{lllllllllll}A & B & \text { C } & \text { D } & \text { E } & \text { F } & \text { G } & \text { H } & \text { I } & \text { J } & \text { K }\end{array}\right]^{\prime}$

onde $A=\Sigma \Sigma \Sigma Y_{1, h}$

Considerando a correspondência entre os termos de 1 a 29 do vetor $Y$ das observaçōes $e$ os valores das colunas $x_{1}, x_{2}, e x$, da matriz $X$, que caracterizam os niveis dos três fatores de cada uma das variáveis $\mathbf{Y}$, pode-se expressar $\mathrm{B}$ da forma seguinte:

$$
\begin{aligned}
B= & -7,262\left(T_{18}\right)-4,391\left(T_{1}+T_{3}+T_{5}+T_{7}\right)-3,631\left(T_{19}\right)-1,000\left(T_{9}+T_{11}+T_{13}+T_{15}\right)+0,000\left(T_{17}+T_{22}+\right. \\
& \left.+T_{23}+T_{24}+T_{25}+T_{26}+T_{27}+T_{28}+T_{29}\right)+1,000\left(T_{10}+T_{12}+T_{14}+T_{16}\right)+3,631\left(T_{20}\right)+4,391\left(T_{2}+T_{4}+\right. \\
& \left.+T_{6}+T_{8}\right)+7,262\left(T_{21}\right)
\end{aligned}
$$

$$
\begin{aligned}
C= & -7,262\left(T_{22}\right)-4,391\left(T_{1}+T_{2}+T_{5}+T_{6}\right)-3,631\left(T_{23}\right)-1,000\left(T_{9}+T_{10}+T_{13}+T_{14}\right)+0,000\left(T_{17}+T_{18}+\right. \\
& \left.+T_{19}+T_{20}+T_{21}+T_{26}+T_{27}+T_{28}+T_{29}\right)+1,000\left(T_{11}+T_{12}+T_{15}+T_{16}\right)+3,631\left(T_{24}\right)+4,391\left(T_{3}+T_{4}+T_{7}+\right. \\
& \left.+T_{8}\right)+7,262\left(T_{25}\right)
\end{aligned}
$$

D é definido de forma correspondente. 


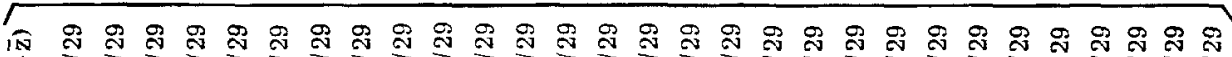

心

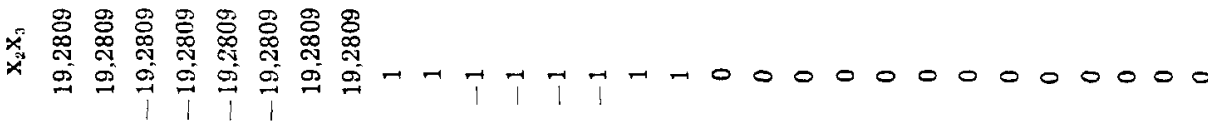

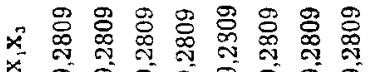

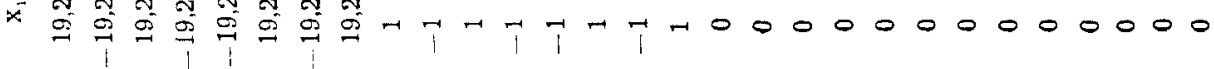

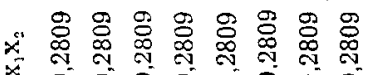

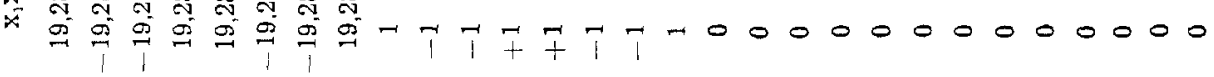

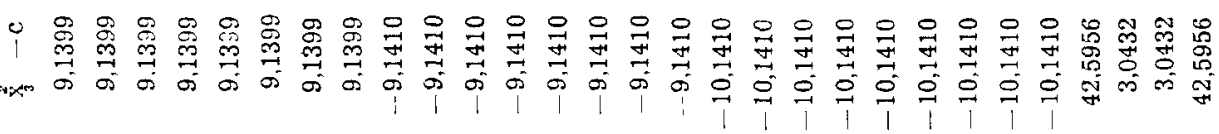

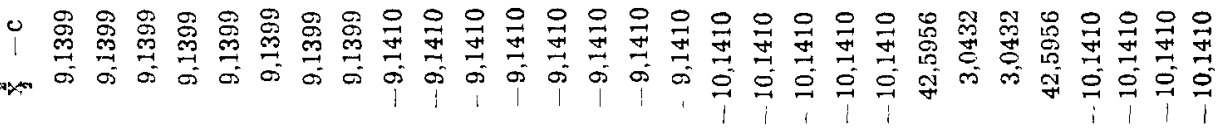

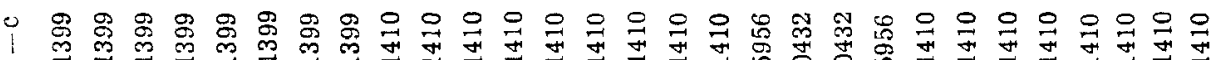

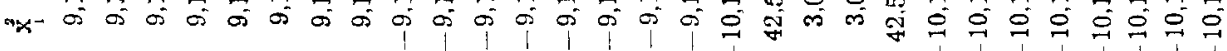

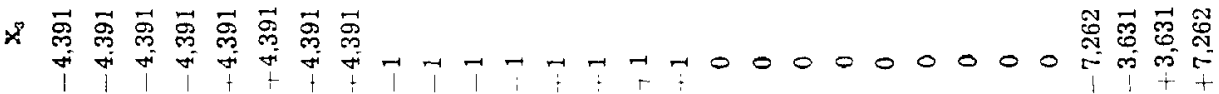

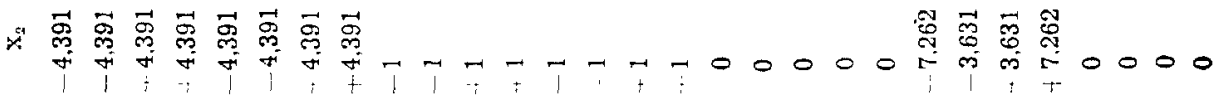

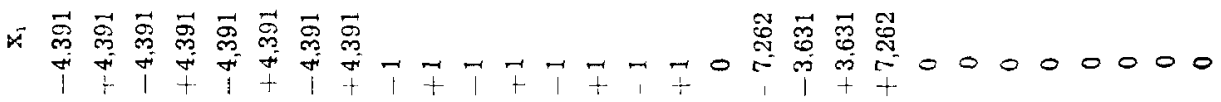

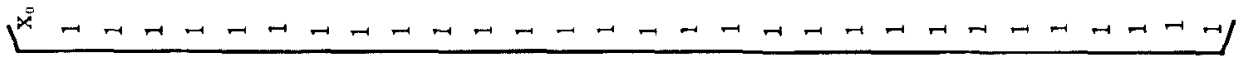
11 密置 


$$
\begin{aligned}
E= & -10,1410\left(T_{17}+T_{22}+\ldots+T_{29}\right)-9,1410\left(T_{9}+T_{10}+\ldots+T_{16}\right)+3,0432\left(T_{19}+T_{20}\right)+9,1399\left(T_{1}+\right. \\
& \left.+T_{2}+\cdots+T_{8}\right)+42,5956\left(T_{18}+T_{21}\right)
\end{aligned}
$$

F e G sāo definidos de forma correspondente.

$$
\begin{aligned}
H= & -19,2809\left(T_{2}+T_{3}+T_{6}+T_{7}\right)-1,0000\left(T_{10}+T_{11}+T_{14}+T_{15}\right)+0,0000\left(T_{17}+T_{18}+T_{19}+\ldots+T_{29}\right)+ \\
& +1,0000\left(T_{9}+T_{12}+T_{13}+T_{16}\right)+19,2809\left(T_{1}+T_{4}+T_{5}+T_{8}\right)
\end{aligned}
$$

I e J são semelhantes.

$K=\frac{13}{29}\left(B_{1}\right)-\frac{16}{29}\left(B_{2}\right)$

onde $\mathbf{B}_{1}$ é a soma dos 16 primeiros termos correspondentes ao bloco $\mathbf{B}_{1} \mathbf{e}, \mathbf{B}_{2}$, a soma dos 13 correspondentes ao bloco $\mathrm{B}_{2}$.

Então:

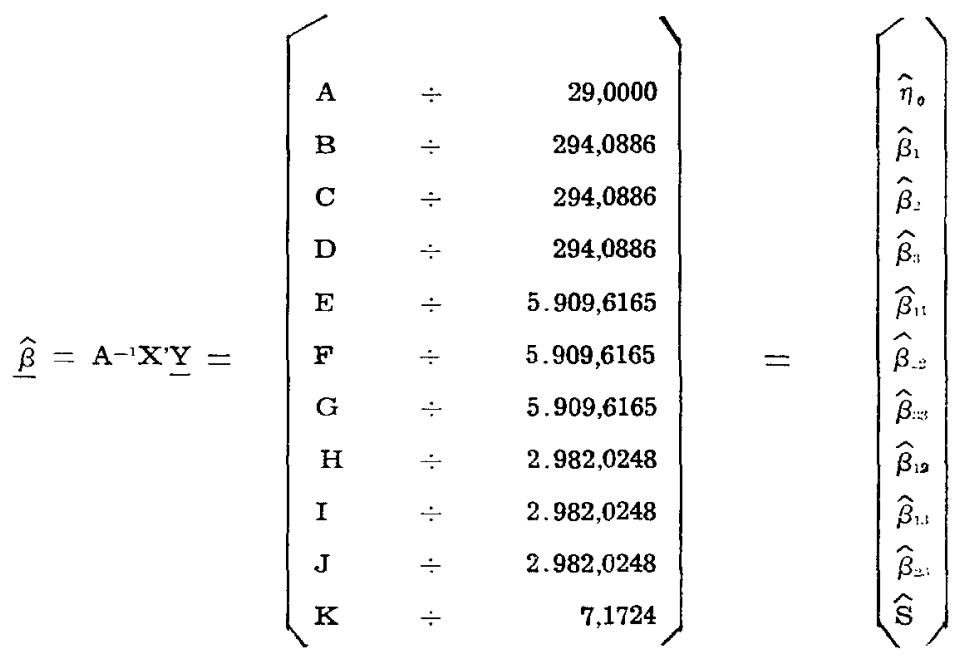

$\hat{Y}=\hat{\eta}_{0}+\hat{\beta}_{1} x_{1}+\hat{\beta}_{2} x_{2}+\hat{\beta}_{3} x_{3}+\hat{\beta}_{11}\left(x_{1}^{2}-c\right)+\hat{\beta}_{22}\left(x_{2}^{2}-c\right)+\hat{\beta}_{33}\left(x_{3}^{2}-c\right)+\hat{\beta}_{12} x_{1} x_{2}+\hat{\beta}_{13} x_{1} x_{3}+\hat{\beta}_{23} x_{2} x_{3}$

onde c, no caso, é igual a 10,14098. Essa é a equação que possibilitará a obtenção dos valores esperados já corrigidos para blocos.

A análise da variância é obtida da forma usual. A parte relativa a blocos pode ser estimada tanto por $\widehat{\mathrm{SK}}$ como pelo cáleulo comum da soma de quadrados de blocos. 
QUADRO 1. - Análise da variância relativa ao delineamento duplo central composto com 29 pontos, em dois blocos

\begin{tabular}{|c|c|c|c|c|c|}
\hline F.V. & S.Q. & G.L. & & Q.M. & $F$ \\
\hline Total & $\Sigma \mathbf{Y}^{2}$ & 29 & & & \\
\hline Média & $\hat{\eta}, \mathbf{A}$ & 1 & & & \\
\hline $\mathrm{N}_{\mathrm{I}}$ & $\widehat{\beta}_{1} \mathrm{~B}$ & 1 & $\widehat{\beta}_{1} \mathbf{B}$ & $\div \quad 1$ & 1 \\
\hline$P_{\mathrm{r}}$ & $\widehat{\beta}_{=} \mathbf{C}$ & 1 & $\hat{\beta}_{2} \mathbf{C}$ & $\div \quad 1$ & 1 \\
\hline $\mathbf{K}_{\mathrm{I}}$ & $\hat{\hat{\beta}}_{:} \mathbf{D}$ & 1 & $\widehat{\beta} . \mathrm{D}$ & $\div \quad 1$ & 1 \\
\hline $\mathrm{N}_{\mathrm{Q}}$ & $\widehat{\beta}_{11} \mathbf{E}$ & 1 & $\hat{\beta}_{11} \mathrm{E}$ & $\div \quad 1$ & 1 \\
\hline $\mathbf{P}_{0}$ & $\widehat{\hat{\beta}}_{\approx} \mathbf{F}$ & 1 & $\hat{\beta}_{\underline{\ldots}} \mathrm{F}$ & $\div \quad 1$ & 1 \\
\hline $\mathbf{K}_{0}$ & $\hat{\beta}_{: 3: G}$ & 1 & $\hat{\beta}_{\mathrm{z}} G$ & $\div \quad 1$ & 1 \\
\hline $\mathbf{N}_{\mathrm{L}} \mathrm{P}_{\mathrm{T}}$ & $\widehat{\hat{\beta}}_{12} \mathrm{H}$ & 1 & $\widehat{\beta}_{12} \mathrm{H}$ & $\div \quad 1$ & 1 \\
\hline $\mathrm{N}_{\mathrm{I}} \mathrm{K}_{\mathrm{L}}$ & $\hat{\boldsymbol{\beta}}_{13} \mathbf{I}$ & 1 & $\hat{\beta}_{13} \mathrm{I}$ & $\div \quad 1$ & 1 \\
\hline $\mathrm{P}_{i} \mathrm{~K}_{\mathrm{I}}$ & $\hat{\beta}_{m,} J$ & 1 & $\hat{\beta}_{m_{3},} J$ & $\div$ & 1 \\
\hline Blocos & $\mathrm{B}_{1}^{2} / 16 \quad-\mathrm{B}_{2}^{2} / 13-\mathrm{A}^{2} / 29$ & 1 & BIs & $\div$ & 1 \\
\hline Residuo & Total - outros $=R$ & 18 & $\mathrm{R}$ & $\div 18$ & 8 \\
\hline
\end{tabular}

Os testes $\mathrm{F}$ são feitos de cada termo em relaçāo ao QM resíduo, sendo $\mathrm{F}$ procurado nas tabelas com 1 e 18 graus de liberdade.

$$
\begin{array}{lll}
V \hat{\eta}_{0}=\frac{\sigma^{2}}{29} & \widehat{V} \hat{\eta}_{0}=s^{2} / 29 \\
v \hat{\beta}_{i}=\frac{\sigma^{2}}{294,0886} & \hat{V} \hat{\beta}_{i}=\frac{s^{2}}{294,0886}, i=1,2,3 \\
V \hat{\beta}_{i i}=\frac{\sigma^{2}}{5909,6165} & \hat{V}_{i i}=\frac{s^{2}}{5909,6165}, i=1,2,3 \\
V \hat{\beta}_{i j}=\frac{\sigma^{2}}{2982,0248} & \hat{V} \hat{\beta}_{i j}=\frac{s^{2}}{2982,0248}, i, j=1,2,3
\end{array}
$$

Para a análise econômica, deve-se ter e expressão de $\widehat{\hat{\mathrm{Y}}}$ em termos dos níveis $\mathrm{X}_{\mathrm{i}}$ originais.

$$
\begin{aligned}
\hat{Y}= & \widehat{\eta}_{0}+\hat{\beta}_{1}\left(X_{1}-7,262\right)+\widehat{\beta}_{2}\left(X_{2}-7,262\right)+\hat{\beta}_{3}\left(X_{3}-7,262\right)+\hat{\beta}_{11}\left[\left(X_{1}-7,262\right)^{2}-c\right]+\hat{\beta}_{22}\left[\left(X_{2}-7,262\right)^{2}-c\right]+ \\
& +\widehat{\beta}_{33}\left[\left(X_{3}-7,262\right)^{2}-c\right]+\widehat{\beta}_{12}\left[\left(X_{1}-7,262\right)\left(X_{2}-7,262\right)\right]+\hat{\beta}_{13}\left[\left(X_{1}-7,262\right)\left(X_{3}-7,262\right)\right]+\widehat{\beta}_{23}\left[\left(X_{2}-\right.\right. \\
& \left.-7,262)\left(X_{3}-7,262\right)\right]
\end{aligned}
$$


Simplificanaio, chega-se a:

$\hat{Y}=\hat{\beta}_{0}+\hat{\beta}_{1} X_{1}+\hat{\beta}_{2} X_{2}+\hat{\beta}_{3} X_{3}+\widehat{\beta}_{11} X_{1}^{2}+\widehat{\beta}_{22} X_{2}^{2}+\hat{\beta}_{33} X_{3}^{2}+\hat{\beta}_{12} X_{1} X_{2}+\hat{\beta}_{13} X_{1} X_{3}+\widehat{\beta}_{23} X_{2} X_{3}$

onde:

$\hat{\beta}_{0}=\hat{\eta}_{0}-7,262\left(\hat{\beta}_{1}+\hat{\beta}_{2}+\widehat{\beta}_{3}\right)+42.596\left(\hat{\beta}_{11}+\hat{\beta}_{22}+\widehat{\beta}_{33}\right)+52,737\left(\hat{\beta}_{12}+\hat{\beta}_{13}+\hat{\beta}_{23}\right)$

$\hat{\beta}_{i}=\hat{\beta}_{1}-14.524 \hat{\beta}_{11}-7.262\left(\hat{\beta}_{12}+\hat{\beta}_{13}\right)$

$\widehat{\beta}_{2}=\widehat{\beta}_{2}-14,524 \widehat{\beta}_{22}-7,262\left(\widehat{\beta}_{12}+\widehat{\beta}_{23}\right)$

$\widehat{\beta}_{3}=\widehat{\beta}_{3}-14.524 \widehat{\beta}_{33}-7.262\left(\widehat{\beta}_{13}+\widehat{\beta}_{23}\right)$

Exemplo - Envolvendo esse último delineamento, é apresentado um exemplo.

Os dados de produçāo encontram-se na páoina 231; estāo assinalados os tratamentos com sua numeração de 1 a 29 , o vetor $\underline{Y}$ das observaçôes, com a especificaçāo dos nuejs dos tratamentos. e suas respectivas produçōes. São dados, na mesma página. os valores esperados para o vetor $\underline{Y}$, usando o modelo quadratico desenvolvido no trabalho. $O s$, tratamentos 1 a 16 constituem o primeiro bloco.

Calculando na forma apresentada às páginas 226 e 228, foram obtidos os elaneitos do vetor $\mathrm{X}^{\prime} \underline{\mathrm{Y}}$, como se segue:

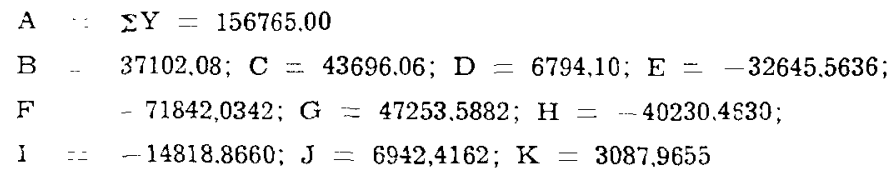

Com esses valores, será calculado $\widehat{\beta}=A^{-1} \mathbf{X}^{\prime} \underline{Y}$,

$\hat{\beta}=\mathrm{A}^{-1} \mathbf{X} \underline{\mathbf{Y}}=$

$\left[\begin{array}{c}\hat{\eta}_{0} \\ \hat{\beta}_{1} \\ \hat{\beta}_{2} \\ \hat{\beta} \\ \hat{\beta}_{13} \\ \hat{\beta}_{2: 2} \\ \hat{\beta}_{1} \\ \hat{\beta}_{12} \\ \hat{\beta}_{13} \\ \hat{\beta}_{21} \\ \hat{\mathrm{S}}\end{array}\right]$

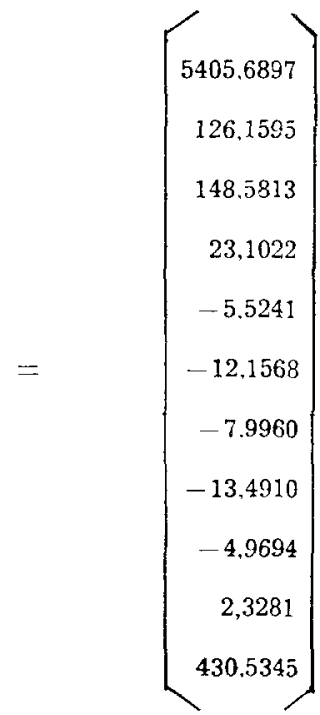

A análise da variância encontra-se no quadro 2 


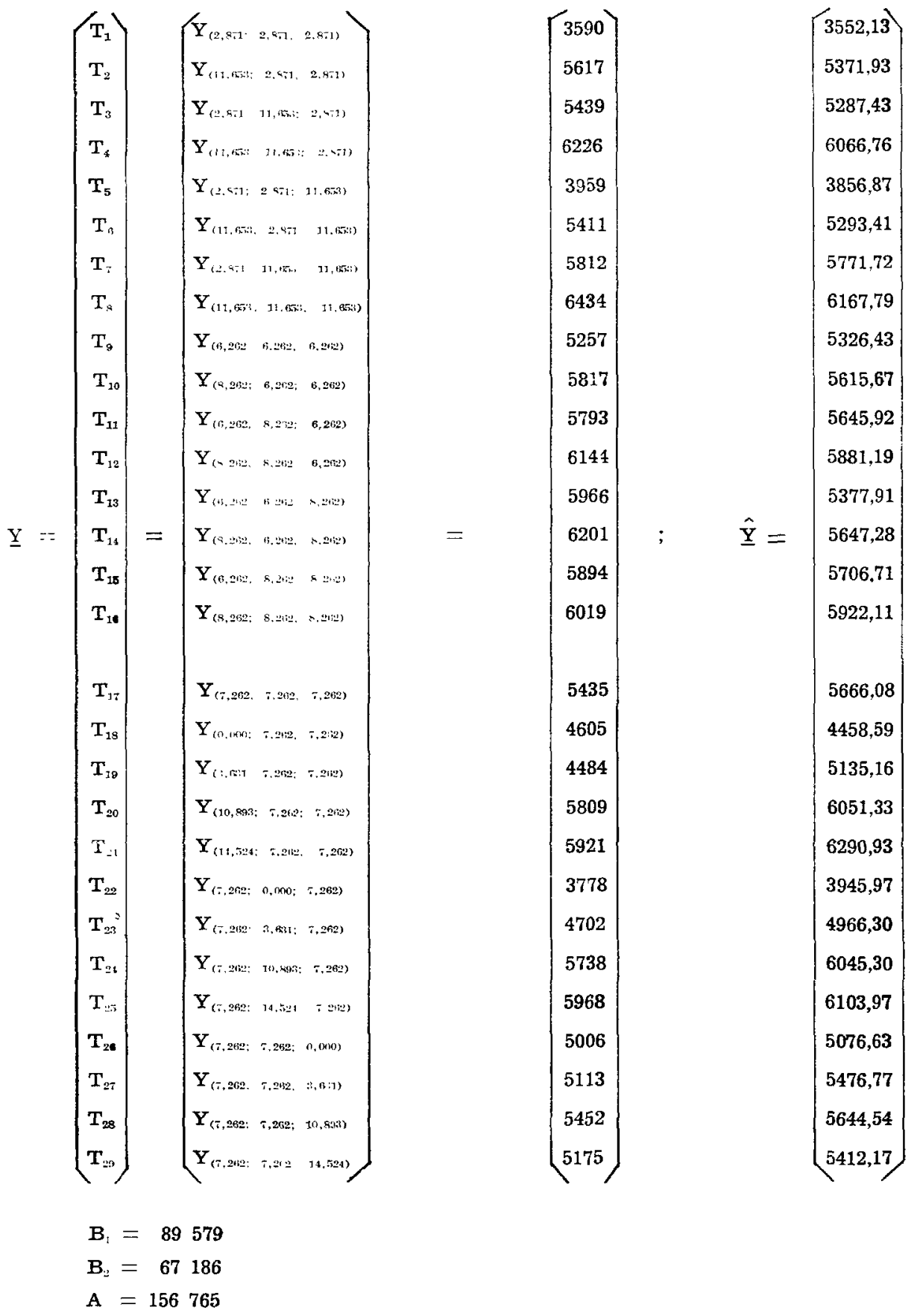


QUADRO 2. - Análise da Variância dos Dados do Exemplo

\begin{tabular}{|c|c|c|c|c|c|}
\hline & & S.Q. & G.L. & Q.M. & $\mathrm{F}$ \\
\hline Total & $\Sigma Y^{i}$ & 862922883.00 & 29 & & \\
\hline Média & $\hat{\eta}_{1}, \mathrm{~A}$ & 847422945.80 & 1 & & \\
\hline $\mathrm{N}_{\mathrm{I}}$ & $\widehat{\beta}_{1} B$ & 4680779,86 & 1 & 4680779,86 & $99,57^{* *}$ \\
\hline$P_{i}$ & $\widehat{\beta}_{C} \mathrm{C}$ & 6492417.40 & 1 & 6492417,40 & $138,10^{* *}$ \\
\hline $\mathrm{K}_{\mathrm{r}}$ & $\widehat{\beta}, D$ & 155958,66 & 1 & 156958,66 & 3,34 \\
\hline $\mathrm{N}_{12}$ & $\hat{\beta}_{11} \mathrm{E}$ & 180337,33 & 1 & 180337,36 & 3,84 \\
\hline $\mathbf{P}_{Q}$ & $\widehat{\beta}_{-F}$ & 873369,24 & 1 & 873369,24 & $18,58^{* *}$ \\
\hline $\mathrm{K}_{\mathrm{U}}$ & $\widehat{\beta} . G$ & 377839.69 & 1 & 377838,69 & $8,04^{* *}$ \\
\hline$N_{\mathrm{t}} P_{\mathrm{t}}$ & $\widehat{\beta}_{, 2} \mathrm{H}$ & 542749,18 & 1 & 542749,18 & $11,54^{* *}$ \\
\hline $\mathrm{N}_{\mathrm{f}}, \mathrm{K}_{\mathrm{I}}$ & $\hat{\beta}, I$ & 73649.87 & 1 & 73640,87 & 1,57 \\
\hline $\mathrm{P}_{\mathrm{L}} \mathbf{K}_{\mathrm{L}}$ & $\widehat{\beta}_{\Perp}, J$ & 16162,64 & 1 & 16162,61 & 0,34 \\
\hline Blocos & & 1329472,90 & 1 & 1329472.90 & $28,28 * *$ \\
\hline Residuo & & 846209,40 & 18 & 47011,63 & \\
\hline
\end{tabular}

$\widehat{V} \hat{\beta}_{i}=\frac{s^{2}}{294,0886}=\frac{47011,63}{294,0886}=159,8553$

$\hat{v} \hat{\beta}_{i i}=\frac{s^{2}}{5909,6165}=\frac{47011,63}{5909,6165}=7,9551$

$\widehat{V} \hat{\beta}_{i j}=\frac{s^{2}}{2982,0248}=\frac{47011,63}{2982,0248}=15,7650$

A equação, nas variáveis originais, vem a seguir:

$$
\begin{aligned}
\widehat{Y}= & 1298,2680+340,4509 X_{1}+406,2116 X_{2}+158,4172 X_{3}-5,5241 X_{1}^{2}-12,1568 X_{2}^{2}-7,9960 X_{3}^{2}- \\
& -13,4910 X_{1} X_{2}-4,9694 X_{1} X_{3}+2,3281 X_{2} X_{3}
\end{aligned}
$$

Os valores esperados foram calculados a partir dessa equação, encontrando-se na última coluna da página 231.

\section{EFICIENCIA DO DELINEAMENTO DUPLO CENTRAL COMPOSTO}

Usando o critério de Box \& Wilson (2) para a avaliação da eficiência do delineamento duplo central composto, em relação ao fatorial $3 \times 3 \times 3$, na estimação dos coeficientes $\beta$, tem-se:

a) Delineamento duplo central composto Não Ortogonal (dcc)

$$
\begin{aligned}
& E f\left(\hat{\beta}_{i}\right) \quad\left\{\frac{d c c}{3 \times 3 \times 3}=\frac{\left(\sigma^{2} / 18\right)\left[1 /(\sqrt{3 / 2})^{2}\right] 27}{\left(\sigma^{2} / 50\right)\left[1 /(\sqrt{29 / 50})^{2}\right] 29}=1,000\right. \\
& E f\left(\hat{\beta}_{i i}\right)\left\{\frac{d c c}{3 \times 3 \times 3}\right\}=\frac{\left(\sigma^{2} / 6\right)(2 / 3)(2 / 3) 27}{0,0214 \sigma^{2}\left[1 /(\sqrt{29 / 50})^{2}\right]\left[1 /(\sqrt{29 / 50})^{2}\right] 29}=1,084 \\
& E f\left(\hat{\beta}_{i j}\right)\left\{\frac{d c c}{3 \times 3 \times 3}\right\}=\frac{\left(\sigma^{2} / 12\right)\left[1 /(\sqrt{3 / 2})^{2}\right]\left[1 /(\sqrt{3 / 2})^{2}\right] 27}{\left.\left(\sigma^{2} / 136\right)[1 / \sqrt{29 / 50})^{2}\right]\left[1 /(\sqrt{29 / 50})^{2}\right] 29}=1,577
\end{aligned}
$$


b) Delineamento duplo central composto ortogonal (deco)

$$
\begin{aligned}
& E f\left(\hat{\beta}_{i}\right) \quad\left\{\frac{d c c o}{3 \times 3 \times 3}\right\}=\frac{\left(\sigma^{2} / 18\right)(2 / 3) 27}{\left(\sigma^{2} / 62,8010\right)(62,8010 / 29) 29}=1,000 \\
& E f\left(\hat{\beta}_{i i}\right)\left\{\frac{d c c o}{3 \times 3 \times 3}\right\}=\frac{\left(\sigma^{2} / 6\right)(2 / 3)(2 / 3) 27}{\left(\sigma^{2} / 176,7623\right)(62,801 / 29)(62,801 / 29) 29}=2,599 \\
& E f\left(\hat{\beta}_{i j}\right)\left\{\frac{d c c o}{3 \times 3 \times 3}\right\}=\frac{\left(\sigma^{2} / 12\right)(2 / 3)(2 / 3) 27}{\left(\sigma^{2} / 136\right)(62,8010 / 29)(62,8010 / 29) 29}=1,000
\end{aligned}
$$

c) Delineamento duplo central composto ortogonal, em dois blocos (dccob)

$$
\begin{aligned}
& E f\left(\hat{\beta}_{i}\right) \quad\left\{\frac{d c c o b}{3 \times 3 \times 3}\right\}=\frac{\left(\sigma^{2} / 18\right)(2 / 3) 27}{\left(\sigma^{2} / 294.0886\right)(294,0886 / 29) 29}=1,000 \\
& E f\left(\hat{\beta}_{i j}\right)\left\{\frac{d c c o b}{3 \times 3 \times 3}\right\}=\frac{\left(\sigma^{2} / 6\right)(2 / 3)^{2} 27}{\left(\sigma^{2} / 5909,6165\right)(294,0886 / 29)^{2} 29}=3,96,3 \\
& E f\left(\hat{\beta}_{i j}\right)\left\{\frac{d c c o b}{3 \times 3 \times 3}\right\}=\frac{\left(\sigma^{2} / 12\right)(2 / 3)^{2} 27}{\left(\sigma^{2} / 2982,0248\right)(294,0886 / 29)^{2} 29}=1,000
\end{aligned}
$$

\section{CONCLUSÃO}

Para o sucesso de programas que visem ao estudo de macronutrientes, com vistas à recomendação final e econômica das dosagens de nutrientes, é de grande importância - uso de delineamentos com um número razoável de níveis e que possibilitem uma análise econômica fảcì e expedita.

o delineamento proposto para o estudo de três fatores, com cinco níveis para o năo-ortogonal e com nove níveis tanto para o ortogonal como para o ortogonal dividido em dois blocos, revela propriedade altamente positiva quando comparado com - clássico $3 \times 3 \times 3$ : possibilita a estimação mais eficiente dos coeficientes quadráticos. Tais coeficientes são diretamente responsáveis pela curvatura da funçāo; como essa curvatura é a maior responsável pela localizaçāo do ponto ótimo econômico, conclui-se que esses delineamentos são superiores ao $3 \times 3 \times 3$ não só por possibilitar o estudo de três fatores com maior número de niveis, como por estimar os coeficientes quadráticos com variância menor que as do $3 \times 3 \times 3$.

\section{DOUBLE CENTRAL COMPOSITE DESIGN WITH 29 POINTS}

\section{SUMMARY}

The double central composite design with 29 points represents an extension of the central composite design and was developed for the study of three factors in more than three levels.

Essentially it is composed of two $2^{3}$ factorials (levels 1 and B), of two stars (levels $\alpha$ and $2 \alpha$ ) and of one central point.

In the present paper the origin of the design, the double central composite completely randomized not orthogonal in 5 levels $(-2,-1,0,+1$ and +2$)$, the completely randomized design, orthogonal, in nine levels $(-3.02 ;-2.00 ;-1.51 ;-1.00 ; 0.00$; 
$+1.00 ;+1.51 ;+2.00 ;+3.02)$ and the orthcgonal design divided in two blocks (levels $-7.262 ;-4.391 ;-3.631 ;-1.000 ; 0.000 ;+1.000 ;+3.631 ;+4.391$ and +7.262 ) are shown.

One example of the late design is presented with its correspondent analysis.

According with Box and Wilson criterion, this last design is more efficient than the $3 \times 3 \times 3$ factorial.

They can be used in fertilizer programs aiming the most efficient economical recommendation of dosages and in other areas of scientific research in which we want to evaluate the surface response equations and its extremum properties looking at the maximum response.

\section{LITERATURA CITADA}

1. AlvareZ, R.; SEGalla, A. L.; WUTKE, A. C. P. \& FREIRE, E. S. Adubação da cana-de-açúcar. VIII - Adubação mineral em solos massapé-salmourāo (1957-58). Bragantia, Campinas, 22:657-675, 1963.

2. BOX, G. E. P. \& WILSON, K. B. On the experimental attainment of optimum conditions. J. R. statist. Soc., B, 13:1-45, 1951.

3. CONAGIN, A. \& JORGE, J. de P. N. Delineamentos (1/5) (5). Bragantia, Campinas, 36:23-58, 1977.

4.

\& VENTURINI, W. R. Delineamentos experimentais utilizáveis na experimentaçāo de campo. In: REYNAERT, E. E., ed. La investigación de fertilidad de suelos para la producción en la zona templada. Montevideo, IICA Zona Sur, 1969. p.183-201.

5. FUZATTO, M. G.; VENTURINI, W. R. \& CAVALERI, P. A. Estudo técnico-econômico da adubação do algodoeiro no Estado de São Paulo. Campinas, Instituto Agronômico, 1970. 15p. (Projeto BNDE/ANDA/CIA, 1)

6. IGUE, T.; MASCARENHAS, H. A. A. \& MIYASAKA, S. Estudo comparativo dos métodos de Mitscherlich e do trinômio do segundo grau, na determinação das doses mais econômicas de fertilizantes, na adubaçāo do feijoeiro (Phaseolus vulgaris L.). C-mpinas, Instituto Agronômico, 1971. 15p. (Projeto BNDE/ ANDA/CIA, 4)

7. MALAVOLTA, E. et alii. A diagnose foliar na cana-de-açúcar: IV. Piracicaba, 1963. 47p.

8. MIRANDA, L. E. C. de \& MIRANDA, L. T. de. Adubação do milho. IV - Estudo econômico de adubação do milho no Estado de São Paulo. Campinas, Instituto Agronômico, 1971. 19p. (Projeto BNDE/ANDA/CIA, 14)

9. MIRANDA, L. T. de. Resultados de experimentos de adubação e sugestōes para a interpretação baseada na análise química de solo. In: Cultura e adubação do milho: São Paulo, Instituto Brasileiro de Potassa, 1966. p.451-472.

10. MYERS, R. H. Response surface met: odology. Boston, Allyn and Bacon, 1971. 243p.

11. PIMENTEL GOMES, F. \& CAMPOS, H. de. Resultados de ensaios da adubação. In: Cultura e adubação do milho. São Paulo, Instituto Brasileiro de Potassa, 1966. p.429-449.

12. RICHARDSON, H. L. Field experiment programmes. Outl. Agric., 1:87-94, 1956.

13. STRAUSS, E. Experimentos de adubação na zona caravieira de Pernambuco. In: Reuniāo Brasileira de Ciências do Solo, 3., 1951. Anais. p.336-446. 
CONAGIN \& JORGE

14. TELLA, R. de; CANECCHIO FILHO, V.; ROCHA, J. L. V.; FREIRE, E. S. \& IGUE, T. Experiências de adubação do amendoim. Campinas, Instituto Agronômico, 1971. 8p. (Projeto BNDE/ANDA/CIA 2)

15. VIEIRA, S.; ARRUDA, H. V. \& HOFFMANN, R. Estudo comparativo de três funçōes na análise econométrica de experimentos de adubação. Piracicaba, Convênio ESCO-MA/ESALQ-USP, 1971. 111p.

16. VOSS, R. \& PESEK, J. T. Yield of corn as affected by fertilizer rates and environmental factors. Agron. J., 59:567-572, 1967. 